\title{
FPGA-based Accelerators for Parallel Data Sort
}

\author{
Valery Sklyarov ${ }^{1}$, Iouliia Skliarova ${ }^{2}$, Alexander Sudnitson ${ }^{3}$, \\ ${ }^{1-2}$ University of Aveiro/IEETA, Portugal, ${ }^{3}$ Tallinn University of Technology, Estonia
}

\begin{abstract}
The paper is dedicated to parallel data sort based on sorting networks. The proposed methods and circuits have the following characteristics: 1) using two-level parallel comparators in even-odd transition networks with feedback to a register keeping input/intermediate data; 2) parallel merging of many sorted sequences; 3) using even-odd transition networks built from other sorting networks; 4) rational reuse of comparators in different types of networks, namely even-odd transition and for discovering maximum/minimum values. The experiments in FPGA, which were done for up to $16 \times 2^{20} 32$-bit data items, demonstrate very good results (as fast as 3-5 ns per data item).
\end{abstract}

Keywords-Merging, performance analysis reconfigurable architectures, sorting.

\section{INTRODUCTION}

Using and taking advantage of hardware accelerators have a long tradition in data processing essential for a vast variety of computational systems. Among numerous problems that need to be solved, sorting is one of the most important [1], [2]. Since it is a time-consuming task for large volumes of data, speed-up is greatly required for many practical applications. A number of recent research activities are targeted to the potential of advanced hardware accelerators, which are analyzed in detail in [3]. Notable results have been achieved through applying parallelism, pipelining, non-sequential circuits, and other techniques and building specialized blocks in hardware. A special attention has been paid to such competitive implementation platforms as: field-programmable gate arrays - FPGAs (e.g., [3]-[7]), graphics processing units GPUs (e.g., [4], [8]-[13]), and multi-core central processing units - CPUs (e.g., [14], [15]). Although different methods have been used in referenced above and other implementations, they can be characterized by a number of common features, such as applying broad parallelism mainly based on different types of sorting networks, minimizing the depth of networks to decrease either the number of clock cycles or delays in combinational circuits that form the network components (comparators), reducing the number of comparators to minimize the required hardware resources, and applying various pipelining techniques for potential accelerations.

One of the most important features of FPGA-based circuits is an opportunity to build the entire system that is composed of various components and data sorters can be among them. Optimization of resources for any component permits the same microchip to be used for implementing additional tasks. Thus, either functionality can be extended or released resources can be used for additional needs, such as better testing, verification, and so forth.
Increase of performance is important for many practical applications, especially for real-time embedded systems. Significant speed-up can be achieved if hardware circuits are used as accelerators for general-purpose and applicationspecific software. A number of comparisons (FPGA vs. multicore CPU, FPGA vs. GPU, FPGA vs. DSP) can be found in [3], [7], [16]-[18]. Existing extendable middleware frameworks, such as VForce [19], permit the same application code to be run in software or in application-specific hardware supporting calls to both FPGAs and GPUs and requiring no changes in user code (results on systems with NVIDIA Tesla GPUs and Xilinx FPGAs are presented in [19]). Thus, application-specific designs can be linked with generalpurpose systems. FPGAs became beneficial for more and more cases mainly due to inherent configurability and relatively cheap development costs. Generally, FPGA-based systems are more redundant and slow compared to CPUs and GPUs. However, they allow creating operations and blocks that are indeed required for particular applications. For example, in parallel sorting, the size of operands can be customized and we can combine reasonably combinational and sequential circuits. Besides, multi-core CPUs and hardware accelerators can be implemented on the same microchip, such as Zynq-7000 from Xilinx [20]. Our experience has shown that the best FPGA-based implementations should be as much regular as possible avoiding complicated routing procedure. Design of such regular circuits for data sort is the main target of this paper.

The remainder of this paper contains seven sections. Section II exposes general ideas for efficient parallel data sort. Section III discusses the related work. FPGA-based implementation and detailed analysis of basic components is done in Section IV. Merging of sorted subsequences for large datasets is described in Section V. Section VI suggests potential improvements to the suggested methods. Section VII discusses implementation details and the results of numerous experiments and comparisons. The conclusion is given in Section VIII.

\section{GENERAL IDEAS}

The proposed method for parallel data sort includes three or less basic steps:

- At the first step, the given set of data is decomposed into subsets that are individually sorted. The size of each subset is limited by FPGA resources, which will be analyzed in subsequent sections.

- At the second step, several subsets are joined to form bigger sorted subsets. Once again, the size of any bigger subset is limited by FPGA resources. 
- At the third step, the subsets are merged to produce the final sorted set.

Suppose we need to sort $N M$-bit data. Figure 1a depicts a very regular circuit which includes cascaded even and odd comparators that are invoked sequentially while there is at least one data swap. Figure $1 \mathrm{~b}$ demonstrates an example for $M=4, N=8$ and the following set of data: $14,15,0,12,4,8$, 1,9 . The circuit is the same as even-odd transition network (see Fig. 2 for the example considered above), but two-level comparators (N/2 comparators at the first level and $N / 2-1$ comparators at the second level) are connected to the register $R$ and multi-level comparisons shown in Fig. 2 are executed sequentially until there are no swaps in all $N-1$ comparators. In the best case (for sorted sequence) the result is ready immediately and in the worst case the result is ready after $N / 2$ steps (clock cycles that control the register). Thus, the minimum delay is 1 clock cycle and the maximum delay is N/2 clock cycles for sorting N M-bit data items.
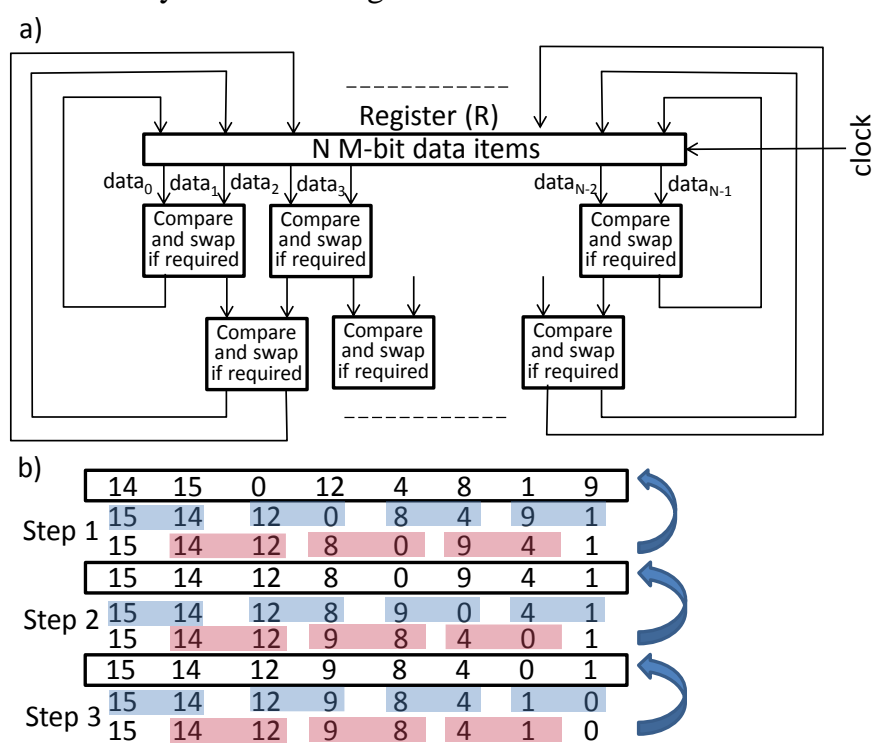

Fig. 1. Sorting network with sequentially invoked even-odd comparators (a); an example (b).

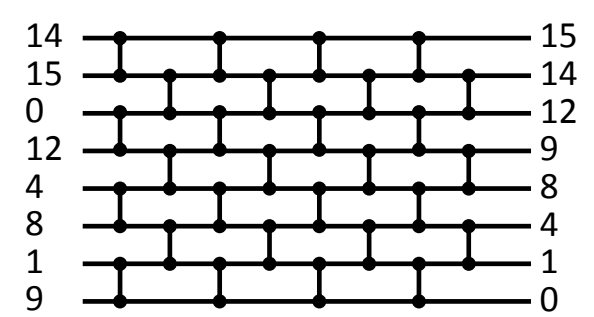

Fig. 2. Even-odd sorting network for the example in Fig. $1 b$.

Let us list basic features of the circuit in Fig. 1a:

- The circuit is very regular and does not require complex interconnections between the elements (the comparators);

- The number of comparators is $N-1$ and they can be implemented within even low-cost FPGAs for relatively large numbers of $N$ (the details and comparisons will be given later).
Although the circuit is sequential, the performance is comparable with multi-level combinational networks because the latter involve significant delays in multi-level circuits.

At the second step, two subsets from the previous step can be joined applying the following method:

1. Comparing and interchanging two sorted subsequences using the method [21] (this method is also described in [1]).

2. Sorting the produced subsets with the largest and the smallest values. As a result, a bigger sequence will be sorted.

Figure 3 shows an example for the following two sorted subsets: 15, 14, 12, 9, 8, 4, 1, 0 (see Fig. 1b and Fig. 2) and 367, 211, 127, 14, 8, 3, 2, 1. The left hand network in Fig. 3 is composed of $N$ comparators for two $N$-bit sorted sequences and the interchange can be done in one clock cycle because comparisons are applied to non-intersecting elements. Sorting by the circuit in Fig. 1a requires N/2 clock cycles (in the worst case). Assuming two sequential sorts for the smallest and the largest subsets, the total joining time is equal to $N+1$ clock cycles. Note that two sorts can be executed in parallel but it doubles the required hardware resources. More than two sorted subsets can be joined using a very similar technique but the time will grow exponentially. Thus, the number of subsets should be reasonably limited, which will be discussed in the subsequent sections.

At the third step, several sorted subsets will be merged using the method illustrated in Fig. 4. Let $K$ be the number of sorted subsets. Each subset $k(0 \leq k \leq K-1)$ contains $N_{k}$ sorted items and all $K$ subsets are kept in dual-port memory blocks embedded to FPGAs.
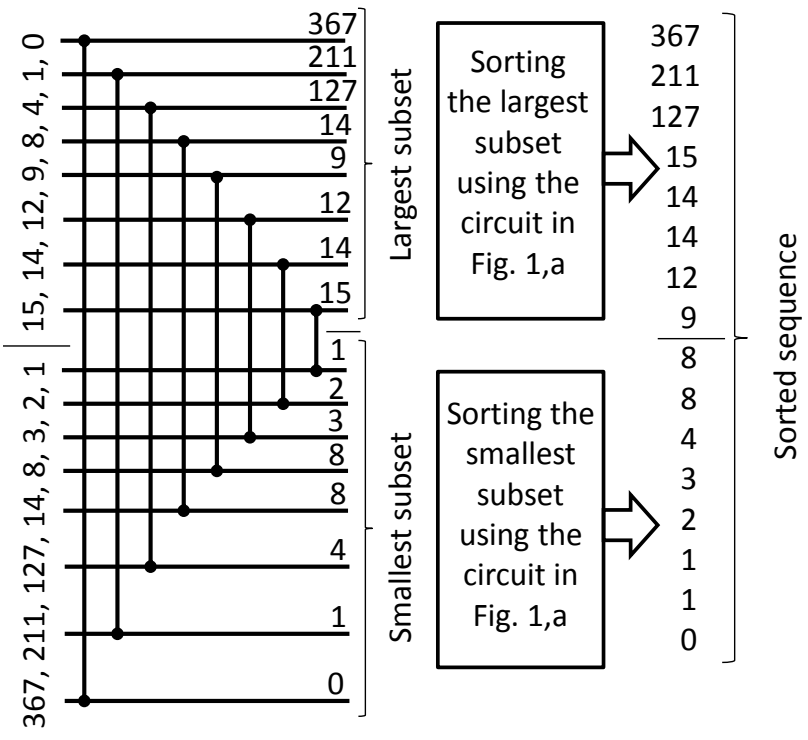

Fig. 3. An example of merging two sorted subsets in one sorted subset.

Each subset has two associated counters pointing respectively to the current maximum and minimum items through the first and the second memory ports. The registers $R_{\max }$ and $R_{\min }$ keep $K$ maximum items (one from each subset) and $K$ minimum items (one from each subset), respectively. The registers and the memory are filled in during forming sorted subsets. Sorting is executed as follows: 
1. Selecting in parallel the maximum value from $R_{\max }$ and the minimum value from $R_{\min }$ with their indices (numbers of associated subsets). Clearly, they are the largest and the smallest values in all subsets.

2. Copying the maximum and the minimum values to the sorted set. At the same time, replacing the maximum and the minimum values in the registers $R_{\max }$ and $R_{\min }$ with the next maximum and the next minimum values from the sorted subsets indicated by the indices from point 1 . Both points 1 and 2 can be completed in one clock cycle. As soon as in the same subset the counter for the minimum value is equal to the counter for the maximum value minus 1 the subset is not used in subsequent steps.

3. The steps 1 and 2 are repeated until the final sorted set is built, which requires $\left(N_{0}+\ldots+N_{K-1}\right) / 2$ clock cycles, i.e., $N / 2$ for $N$ items in all the subsets.

As an example, Fig. 5 presents the first 8 steps (clock cycles) for merging the following four subsets of sorted items: 1) $15,14,12,9,8,4,1,0$; 2) $367,211,127,14,8,3,2,1$; 3) $415,10,9,7,5,3,2,1$; 4) 99, 88, 88, 87, 62, 51, 50, 45.

The circuits, which discover the maximum and the minimum values, can be built as networks shown in Fig. 6 (for $K=16)$.

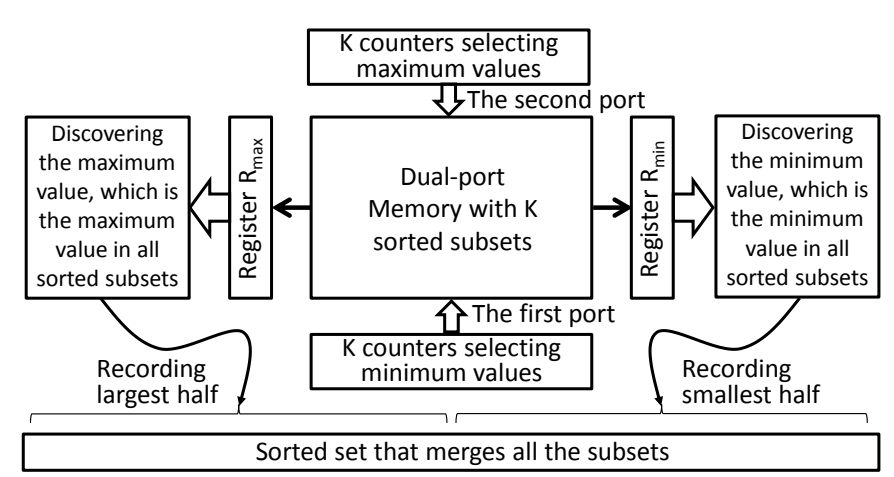

Fig. 4. Merging $K$ sorted subsets.

Since the first and the last steps are executed in different time slots, the first and the second levels of comparators shown in Fig. 1a can be reused in the networks of Fig. 6. It means that two lines of multiplexers are added: the first line selects either the set for sorting or $R_{\max }$; and the second line selects either the first level of comparators (see Fig. 1a) or $R_{\min }$ (note that one additional comparator is needed for $R_{\min }$ ). Thus, the number of additional comparators for $K$ subsets is equal to $2 \times\left(2^{0}+2^{1}+\ldots+K / 4\right)$. For our example in Fig. $6 K / 4=4$ and the number of additional comparators is $2 \times(1+2+4)=14$. The circuit in Fig. 6 is combinational.

5) 15

14

10

$15,14,12,9,8,-4, \ldots, \theta \quad 4$ $367,211,127,14,8,3, z, 1 \rightarrow z$ $415,10,9,7,5,3,2,1 / 2$

$\left(\begin{array}{ccccr}415 & 415,10,9,7,5,3,2,1 & 1 \\ 99 & 99,88,88,87,62,51,50,45 & 45 \\ \text { max } & \text { ONE CLOCK CYCLE } & \text { min }\end{array}\right)$ $99^{\circ}$ $99,88,88,87,62,51,50,45 \quad 45$ $\max \longrightarrow$ ONE CLOCK CYCLE

$04415,367,211,127,99 \quad 2,1,1,1,0$

415

2) $15 \quad 15,14,12,9,8,4,1, \theta$ $\left(\begin{array}{cccr}367 & 367,211,127,14,8,3,2,4 & 1 \\ 10 & 415,10,9,7,5,3,2,4 & 1 \\ 99 & 99,88,88,87,62,51,50,45 & 45 \\ \text { max } & \text { ONE CLOCK CYCLE } & \min \end{array}\right)$

$415,367 \quad 1,0$

3) 15

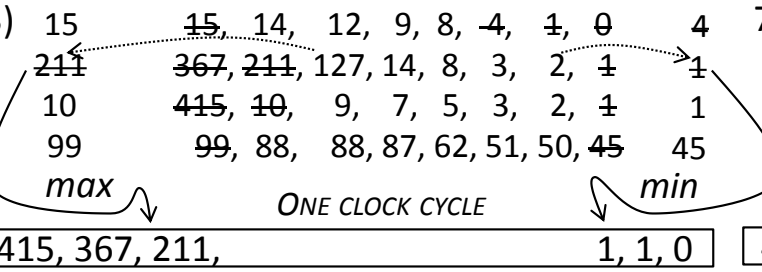

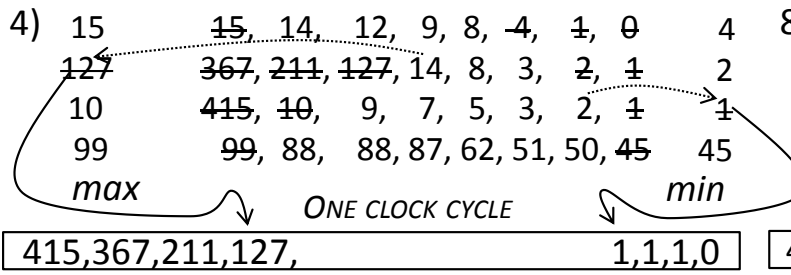

6) $1515,14,12,9,8,-4,4, \theta \quad 4$ $14367,211,127,14,8,3, z, 13$ $10 \quad 415, \ldots, 9,7,5,3, z, 1 / \beta_{z}$ $88 \div 99,88,88,87,62,51,50,4545$ $\max O$ ONE CLOCK CYCLE $\swarrow \min$

$415,367,211,127,99,88, \quad 2,2,1,1,1,0$

7) $15 \quad 15,14,12,9,8,-4,1, \theta \quad \theta$ $14367,211,127,14,8,3, z, 1 \mathrm{~m}_{3}$ $10 \quad 415,10, \ldots 9,7,5,3, z, 1 / 3$ $87 \div 99,88,88,87,62,51,50,4545$ $\max$ ONE CLOCK CYCLE $\min$ $415,367,211,127,99,88,87, \quad 3,2,2,1,1,1,0$

8) $15 \quad 15,14,12,9,8,-4,4, \theta 4$ $14 \quad 367,211,127,14,8,3, z, 1,8$ $10415,10, \ldots, \ldots, 5,3, z, 1 / 3$ $62 \div 99,88,88,87,62,51,50,45 / 45$ $\max$ ONE CLOCK CYCLE $\min$ $415,367,211,127,99,88,87,62 \quad \ldots . .3,3,2,2,1,1,1,0$

Fig. 5. The first 8 steps for merging $K$ sorted subsets: $K=4, N_{0}=N_{1}=N_{2}=N_{3}=8$. 


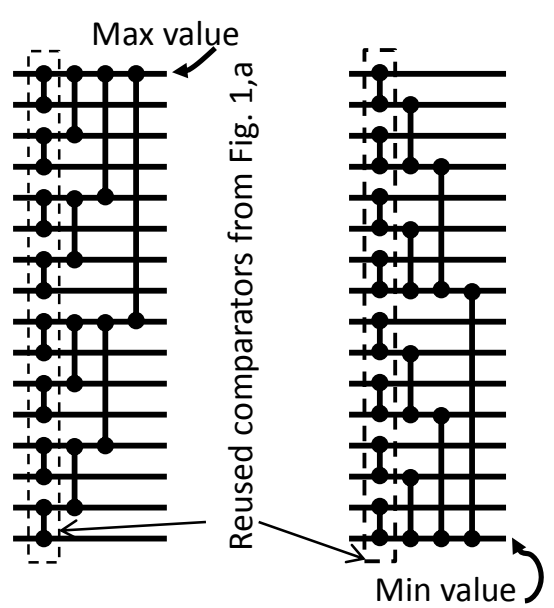

Fig. 6. Discovering the maximum/minimum values in the registers of Fig. 5.

Suppose $N M$-bit items have to be sorted and we will execute just the first and the third steps. At the first step, $K$ sorted subsets are created sequentially. In fact, such subsets can be built in parallel if FPGA resources are sufficient. Any subset $k$ requires $N_{k} / 2$ clock cycles in the worst case. Assuming that all values $N_{0}, \ldots, N_{K-1}$ are equal we need $K \times\left(N_{\mathrm{k}} / 2\right)$ clock cycles for the first step. The third step requires $N / 2$ clock cycles. Thus, the total time is $K \times\left(N_{k} / 2\right)+N / 2=N$ (if all $K$ sorted subsets have equal number of elements) in the worst case. What is very important is the low delay in combinational paths of different circuits, i.e., the minimal potential clock cycle period. Such characteristics will be thoroughly analyzed in real experiments with different FPGA-based circuits. We will also present the results of simulation in software for different datasets.

\section{RELATED WORK}

Since sorting is a core problem in computer science, it has been very well researched over the last five decades [11]. The main direction in this area is aimed at different types of parallel implementations involving sorting networks. In [3], [7] various types of networks were implemented in FPGA and it was shown that even in advanced FPGAs (such as from Virtex-5 family of Xilinx) it is possible to construct combinational networks just up to 64 items, which is undoubtedly non-sufficient. In the subsequent sections we will demonstrate that using the circuits from Fig. 1a, sorters for significantly more items can be built even in low-cost FPGAs. The results of comparisons of different networks [3] (namely bitonic merge and even-odd merge) show that even-odd merge networks require slightly smaller hardware resources. From [1] we can see the minimum number of comparators in different networks. Although even-odd transition networks are not the best [12] (from the point of view of hardware resources and the number of levels) they are very regular. Besides, the two-level comparator structure shown in Fig. 1a cannot be built for bitonic merge and even-odd merge networks. To our knowledge, such networks are among the fastest but they cannot be used in a way considered in the paper. Comparisons of improved bitonic networks proposed in [11] with state-ofthe-art sorting methods (namely quicksort and radixsort) demonstrate advantages of methods [11] and, thus, the results of [3], [7], [11] can be taken for comparison with our technique. Besides, the best networks can be used as components within the proposed circuits and we will demonstrate such an opportunity in subsequent sections. Many other publications [3]-[15] present platform-targeted (multicore CPU, GPU, FPGA) methods. Our approach is more relevant to FPGAs. There are some reasons for that. Current trends for FPGA vendors demonstrate that more and more standard blocks (memories, digital signal processors, multicore CPUs, transceivers, etc.) are accommodated with gate arrays on the same microchips. An example is a dual-core Cortex-A9 ARM processor embedded to Zynq microchips of Xilinx and combined with Artix/Kintex FPGAs [20]. Thus, we can expect that as functionality of individual micro-chips is more and more integrated in ASICs (application-specific integrated circuits) and ASSPs (application-specific standard products), FPGA will contain more and more ASICs and ASSPs functionality implemented as built-in units. We presume that one of such units might be GPU. Thus, the boundary between different platforms will be eliminated [22]. Although our technique is FPGA-targeted, it can be combined with other implementations. Some examples are given below:

- Rapid creation of $K$ sorted subsets, which can be further merged in a single sorted data set in general-purpose computers, multi-core CPU, etc.;

- Using fast bitonic (or some other) networks [11] in Fig. 1a for sorting more than two items at each comparator level (see some particular proposals in Section VI);

- Rational combination of address-based sorting [23] for very large data sets (close to $2^{N}$ ) and the proposed technique for smaller data sets $\left(N<<2^{N}\right)$;

- Sorting $M$-bit data with very big values of $M$, which is easier in FPGAs since many constraints inherent to GPUs and multi-core CPUs can be eliminated (the results of experiments with 128-bit data items, i.e., $M=128$, are given in Section VII);

- Design of fastest sorters with small number of $N$ using just the circuit is Fig. 1a. Such sorters are very valuable for different priority buffers/queues [24];

- Using the results of [19] permitting the same application code to be run in software or in application-specific hardware (FPGA in our case).

\section{FPGA-BASED IMPLEMENTATION AND A THOROUGH COMPARISON OF BASIC COMPONENTS}

In this section, we evaluate the primary components to be used as building blocks for the considered sorters, which are:

- Comparators;

- A two-level sequential circuit shown in Fig. 1a;

- The circuits that discover the maximum and the minimum values in given sets of items (see Fig. 6).

The objective is to get the following parameters for different FPGAs:

- Maximum delay in combinational circuits;

- Maximum attainable clock frequency;

- Required hardware resources. 
Table II demonstrates that sorting 512 32-bit data items in the FPGA xc5vlx110t is completed in 2.1 microseconds $(\mu s)$, which is very fast. Sorting 256 32-bit data items in the lowcost FPGA xc6slx45 is completed in $1.5 \mu \mathrm{s}$. These results were actually verified in hardware (the prototyping boards ML505 and Atlys). The results of synthesis and implementation demonstrate that sorting 1024 32-bit data items in the FPGA xc6vlx240t can be done in $3.4 \mu \mathrm{s}$.

\section{TABLE II}

COMPLEXITIES OF THE CiRCUIT IN Fig. 1A FOR SORTING DifFerent SETS

\begin{tabular}{|c|c|c|}
\hline \multirow{3}{*}{$\begin{array}{l}\text { Xilinx Spartan-3e } \\
\text { xc3s 1200e FPGA }\end{array}$} & $\mathrm{S}_{\mathrm{FPGA}} / \mathrm{S} / \mathrm{F}_{\max }=8,672 / 934 / 73.99$ & $N=16$ \\
\hline & $\mathrm{S}_{\mathrm{FPGA}} / \mathrm{S} / \mathrm{F}_{\max }=8,672 / 3,647 / 70.831$ & $N=64$ \\
\hline & $\mathrm{S}_{\mathrm{FPGA}} / \mathrm{S} / \mathrm{F}_{\max }=8,672 / 7,299 / 66.635$ & $N_{\max }=128$ \\
\hline \multirow{3}{*}{$\begin{array}{l}\text { Xilinx Spartan-6 } \\
\text { xc6slx45 FPGA }\end{array}$} & $\mathrm{S}_{\mathrm{FPGA}} / \mathrm{S} / \mathrm{F}_{\max }=6,822 / 1,425 / 95.033$ & $N=64$ \\
\hline & $\mathrm{S}_{\mathrm{FPGA}} / \mathrm{S} / \mathrm{F}_{\max }=6,822 / 2,899 / 93.182$ & $N=128$ \\
\hline & $\mathrm{S}_{\mathrm{FPGA}} / \mathrm{S} / \mathrm{F}_{\max }=6,822 / 4,506 / 89.841$ & $N_{\max }=256$ \\
\hline \multirow{3}{*}{$\begin{array}{l}\text { Xilinx Virtex-4 } \\
\text { 4VFX12 FPGA }\end{array}$} & $\mathrm{S}_{\mathrm{FPGA}} / \mathrm{S} / \mathrm{F}_{\max }=5,472 / 954 / 123.096$ & $N=16$ \\
\hline & $\mathrm{S}_{\mathrm{FPGA}} / \mathrm{S} / \mathrm{F}_{\max }=5,472 / 1,861 / 116.380$ & $N=32$ \\
\hline & $\mathrm{S}_{\mathrm{FPGA}} / \mathrm{S} / \mathrm{F}_{\max }=5,472 / 3,674 / 112.809$ & $N_{\max }=64$ \\
\hline \multirow{3}{*}{$\begin{array}{l}\text { Xilinx Virtex-5 } \\
\text { xc5vlx110t } \\
\text { FPGA }\end{array}$} & $\mathrm{S}_{\mathrm{FPGA}} / \mathrm{S} / \mathrm{F}_{\max }=17,280 / 1,642 / 134.509$ & $N=64$ \\
\hline & $\mathrm{S}_{\mathrm{FPGA}} / \mathrm{S} / \mathrm{F}_{\max }=17,280 / 6,928 / 131.854$ & $N=256$ \\
\hline & $\mathrm{S}_{\mathrm{FPGA}} / \mathrm{S} / \mathrm{F}_{\max }=17,280 / 12,180 / 124.041$ & $N_{\max }=512$ \\
\hline \multirow{3}{*}{$\begin{array}{l}\text { Xilinx Virtex-6 } \\
\text { xc6vlx240t } \\
\text { FPGA }\end{array}$} & $\mathrm{S}_{\mathrm{FPGA}} / \mathrm{S} / \mathrm{F}_{\max }=37,680 / 1,161 / 180.107$ & $N=64$ \\
\hline & $\mathrm{S}_{\mathrm{FPGA}} / \mathrm{S} / \mathrm{F}_{\max }=37,680 / 11,006 / 147.739$ & $N=512$ \\
\hline & $\mathrm{S}_{\mathrm{FPGA}} / \mathrm{S} / \mathrm{F}_{\max }=37,680 / 21,772 / 154.774$ & $N_{\max }=1024$ \\
\hline \multirow{3}{*}{$\begin{array}{l}\text { Altera Cyclon- } \\
\text { IVe EP4CE115 } \\
\text { FPGA }\end{array}$} & $\mathrm{LE}_{\mathrm{FPGA}} / \mathrm{LE} / \mathrm{F}_{\max }=114,480 / 3,166 / 63.71$ & $N=64$ \\
\hline & $\mathrm{LE}_{\mathrm{FPGA}} / \mathrm{LE} / \mathrm{F}_{\max }=114,480 / 39,564 / 47.61$ & $N=512$ \\
\hline & $\mathrm{LE}_{\mathrm{FPGA}} / \mathrm{LE} / \mathrm{F}_{\max }=114,480 / 87,400 / 36.15$ & $N_{\max }=1024$ \\
\hline \multirow{3}{*}{$\begin{array}{l}\text { Xilinx APSoC } \\
\text { Zynq XC7Z020 }\end{array}$} & $\mathrm{S}_{\mathrm{FPGA} A} / \mathrm{S} / \mathrm{F}_{\max }=13,300 / 1,203 / 189.654$ & $N=64$ \\
\hline & $\mathrm{S}_{\mathrm{FPGA}} / \mathrm{S} / \mathrm{F}_{\max }=13,300 / 2,022 / 184.459$ & $N=128$ \\
\hline & $\mathrm{S}_{\mathrm{FPGA}} / \mathrm{S} / \mathrm{F}_{\max }=13,300 / 4,079 / 183.882$ & $N_{\max }=256$ \\
\hline
\end{tabular}

\section{Discovering the Maximum and the Minimum Items}

In Section II we have explained that since the first and the last steps are executed in different time slots, the first and the second levels of comparators shown in Fig. 1a can be reused in the networks of Fig. 6. Table III gives the results of synthesis and implementation of such circuits in different FPGAs in the following format: the number of occupied FPGA slices (logic elements for Altera) S_LE indicating in parenthesis percentage of S_LE from all the available in particular FPGA slices (logic elements for Altera); the maximum attainable clock frequency $F_{\max }$ in $\mathrm{MHz}$, and the total number of comparators $N_{\text {c. }}$ These data are recorded in the format: S_LE (percentage) $/ F_{\max } / N_{\mathrm{c}}$.

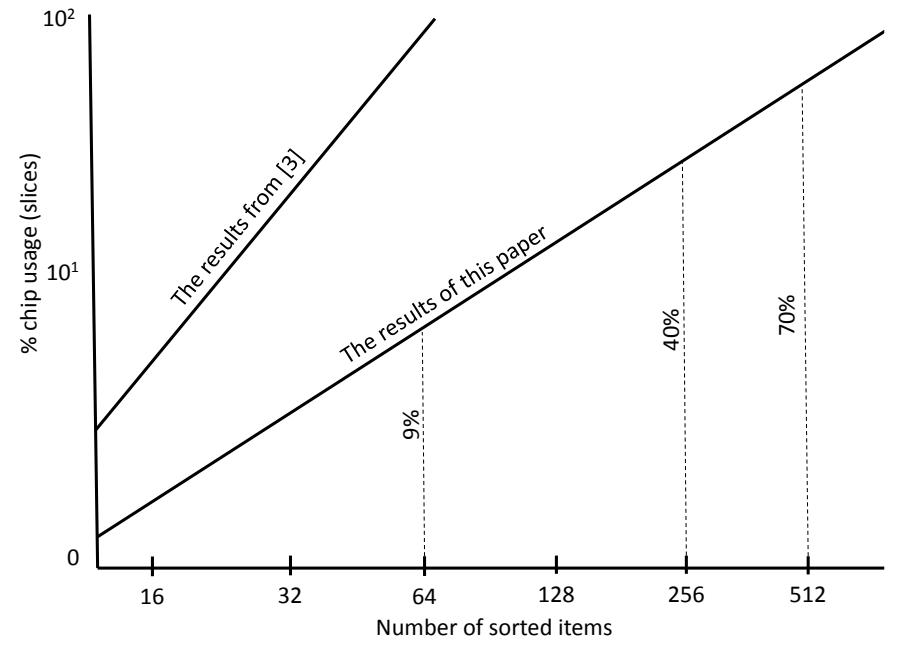

Fig. 7. Comparison of the results with [3].

From Table III we can conclude that resources are very reasonable and enable us to implement the proposed sorters even in low-cost commercial FPGAs. There is some inconsistence in different tables, for example, the number of slices for one comparator is 21 for Spartan-6 FPGA (see Table I). It means that the total number of comparators in the last row of Table III should exceed the available FPGA resources. However, the resources were sufficient and we assume that it happened due to some optimization techniques applied by ISE 14.1 (such as avoiding some redundant signals).

\section{MERGING SORTED SUbSEQUENCES FOR LARGE SCALE DATASETS}

Synthesis and implementation of merging circuits require very long time. That is why we tested in FPGAs just relatively simple circuits (up to $2^{14} 32$-bit data items). At the same time, we modeled the circuits in general-purpose software (in $\mathrm{C}++$ language) and counted the number of FPGA clock cycles. Since the number of actual clock cycles in FPGA and the number of clock cycles obtained from software programs were exactly the same, we decided to present the results of more complicated sorters (up to millions of 32-bit items) just from simulation in software.

Sorting subsets is done in the following $\mathrm{C}++$ fragment, which uses two levels of comparators invoked in a cycle activated as long as the set is not sorted:

TABLE III

COMbining Even-odd Networks ANd Circuits that Find out Minimum/MaXimum Values in Given Subsets

\begin{tabular}{|c|c|c|c|c|c|c|c|}
\hline $\begin{array}{l}\mathrm{N} \\
\text { (32 bits) }\end{array}$ & $\begin{array}{l}\text { Spartan-3 } \\
\text { xc3s1200e }\end{array}$ & $\begin{array}{l}\text { Spartan-6 } \\
\text { xc6s } 1 \times 45\end{array}$ & $\begin{array}{l}\text { Virtex-4 } \\
\text { 4VFX12 }\end{array}$ & $\begin{array}{l}\text { Virtex-5 } \\
\text { xc5vlx110t }\end{array}$ & $\begin{array}{l}\text { Virtex-6 } \\
\text { xc6vlx240t }\end{array}$ & $\begin{array}{l}\text { Zynq } \\
\text { XC7Z020 }\end{array}$ & $\begin{array}{l}\text { Cyclon-IVe } \\
\text { EP4CE115 }\end{array}$ \\
\hline 16 & $\begin{array}{l}2,086(24 \%) / \\
57.183 / 62\end{array}$ & $\begin{array}{l}1,123(16 \%) / \\
82.594 / 62\end{array}$ & $\begin{array}{l}2,106(38 \%) / \\
87.507 / 62\end{array}$ & $\begin{array}{l}1,206(6 \%) / \\
122.869 / 62\end{array}$ & $\begin{array}{l}1,113(2 \%) / \\
180.401 / 62\end{array}$ & $\begin{array}{l}1,225(9 \%) / \\
190.981 / 62\end{array}$ & $\begin{array}{l}2,146(2 \%) / \\
69.9 / 62\end{array}$ \\
\hline 32 & $\begin{array}{l}4,173(48 \%) / \\
56.886 / 126\end{array}$ & $\begin{array}{l}2,304(33 \%) / \\
83.299 / 126\end{array}$ & $\begin{array}{l}4,218(77 \%) / \\
82.390 / 126\end{array}$ & $\begin{array}{l}2,190(12 \%) / \\
117.726 / 126\end{array}$ & $\begin{array}{l}1,838(4 \%) / \\
179.889 / 126\end{array}$ & $\begin{array}{l}1,930(14 \%) / \\
187.337 / 126\end{array}$ & $\begin{array}{l}10,640(9 \%) / \\
46.23 / 126\end{array}$ \\
\hline 64 & $\begin{array}{l}8,329(96 \%) / \\
52.953 / 254\end{array}$ & $\begin{array}{l}4,232(62 \%) / \\
82.802 / 254\end{array}$ & $\begin{array}{l}S>100 \% \text { of } \\
\text { available resources }\end{array}$ & $\begin{array}{l}4,192(24 \%) / \\
112.267 / 254\end{array}$ & $\begin{array}{l}3,705(9 \%) / \\
175.562 / 254\end{array}$ & $\begin{array}{l}3,973(29 \%) / \\
182.749 / 254\end{array}$ & $\begin{array}{l}21,922(19 \%) / \\
40.74 / 254\end{array}$ \\
\hline 128 & $\begin{array}{l}S>100 \% \text { of } \\
\text { available } \\
\text { resources }\end{array}$ & $\begin{array}{l}6,799(99 \%) / \\
79.543 / 510\end{array}$ & $\begin{array}{l}S>100 \% \text { of } \\
\text { available resources }\end{array}$ & $\begin{array}{l}7,964(46 \%) / \\
101.867 / 510\end{array}$ & $\begin{array}{l}7,020(18 \%) / \\
165.741 / 510\end{array}$ & $\begin{array}{l}7,227(54 \%) / \\
174.113 / 510\end{array}$ & $\begin{array}{l}44,077(39 \%) / \\
34.76 / 510\end{array}$ \\
\hline
\end{tabular}


for (int $\mathrm{n}=0 ; \mathrm{n}<$ number of blocks; $\mathrm{n}++$ )

$/ / \mathrm{K}$ = number_of_blocks

\{

do /* exchange flag indicates occurrence of at least of one swapping in parallel even sorter or in parallel_odd_sorter*/

\{ exchānge flag $^{-}$false;

clock cyclest+; /* each iteration is executed in one clock cycle (see Fig. 1a)*/

// simulates the first level of comparators parallel even sorter (word[n], number of dàta in block);

// simulates the second level of comparators parallel odd sorter (word[n], number_of_data_in_block);

while (exchange flag != false);

$\max \operatorname{reg}[\mathrm{n}]=$ word $[\mathrm{n}][0]$;

// register that keeps $\mathrm{K}$ maximum values

min reg $[n]=$ word $[n]$ [number of data in block-1];

// register that keeps $\mathrm{K}$ miñimüm value $\bar{s}$ \}

Merging the sorted subsets is done in the following $\mathrm{C}++$ fragment:

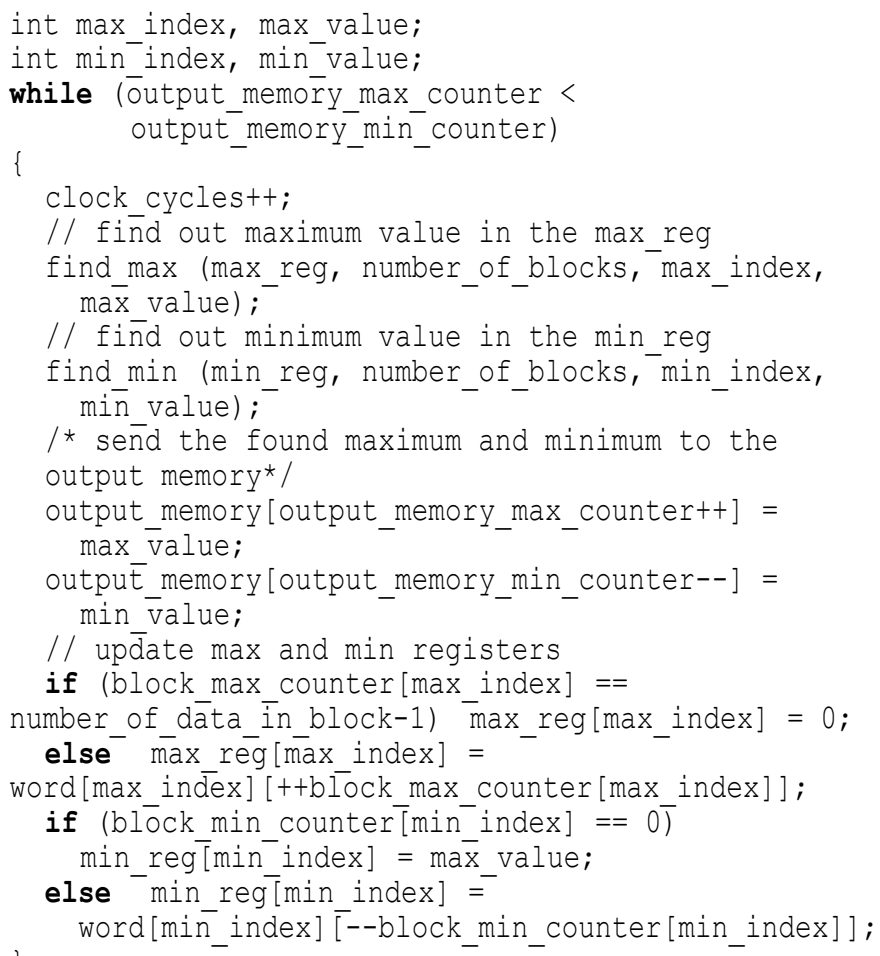

Initial data inside FPGAs were generated randomly and stored in embedded block RAMs in such a way that allows all $N M$-bit data to be recorded to the register in Fig. 1a and to be written back to memory in parallel. For FPGA implementations $K=256$ sorted blocks with 64 32-bit items in each block were taken for merging and producing the final sorted sequence containing $2^{14}$ 32-bit items. The maximum attainable clock frequency for Spartan-6 FPGA was about $80 \mathrm{MHz}$. The results of simulation in software based on two steps (1 and 3) are presented in Table IV for different values of $K$ and $N_{k}$ (assuming all blocks have equal number of data items).
TABLE IV

THE RESUltS OF SimUlation IN SOFTWARE APPLYING STEPS 1 AND 3

\begin{tabular}{|c|c|c|c|c|}
\hline $\begin{array}{l}\text { Number } \\
\text { of } \\
\text { blocks } \\
K\end{array}$ & $\begin{array}{l}\text { Number } \\
\mathrm{N}_{\mathrm{k}} \text { of } \\
\text { data in } \\
\text { block } \\
\left(N_{0}=N_{1}=\right. \\
\left.\ldots=N_{K-1}\right)\end{array}$ & $\begin{array}{l}\text { Total number } \\
N \text { of data } \\
\text { items }\end{array}$ & $\begin{array}{l}\text { Number of } \\
\text { clock } \\
\text { cycles (2- } \\
\text { level } \\
\text { sorting } \\
\text { network) }\end{array}$ & $\begin{array}{l}\text { Number of clock } \\
\text { cycles (2-level } \\
\text { sorting network, } \\
\text { which analyzes } \\
\text { groups of items } \\
\text { with } t=8 \text { ) }\end{array}$ \\
\hline 16 & 16 & 256 & 240 & 176 \\
\hline 32 & 32 & 1,024 & 907 & 670 \\
\hline 64 & 64 & 4,096 & 3,542 & 2,602 \\
\hline 128 & 128 & 16,384 & 14,103 & 10,280 \\
\hline 256 & 256 & 65,536 & 56,394 & 40,894 \\
\hline 512 & 256 & 131,072 & 112,739 & 81,780 \\
\hline 512 & 512 & 262,144 & 226,417 & 163,379 \\
\hline 1,024 & 512 & 524,288 & 452,712 & 326,718 \\
\hline 1,024 & 1,024 & $1,048,576$ & 908,048 & 653,276 \\
\hline 2,048 & 1,024 & $2,097,152$ & $1,815,948$ & $1,306,498$ \\
\hline 2,048 & 2,048 & $4,194,304$ & $3,641,840$ & $2,614,155$ \\
\hline
\end{tabular}

We found that different numbers of $M$ affect mainly the consumed hardware resources and practically do not alter the maximum achievable clock frequency (three different sizes $M=32, M=64$ and $M=128$ were examined). The results of simulation in software based on all three steps do not give any advantage and the performance is even decreased. So, the step 2 was removed. That is why in all future implementations just the first and the third steps were used.

\section{POTENTIAL IMPROVEMENTS}

Addition speed-up of the circuit from Fig. 1a can be achieved if comparators are replaced with sorting networks processing more than two items simultaneously. Let us consider the circuit in Fig. 8a where data items in a given subset are divided in $G$-element groups. Data within each group are sorted using networks. As a result, instead of evenodd transition network for pairs of items we consider even-odd transition network for $t$-element groups of items. Figure $8 \mathrm{~b}$ gives an example of sorting 16 data items divided in two 8-element groups (i.e., $G=2, t=8$ ). Such improvements permit additional acceleration of sorting in $\log _{2}$ t times (in 3 times for our example). However, FPGA resources will also be increased significantly. Indeed, for our example $(t=8)$ the best sorting networks contain 19 comparators [1] and the number of slices required for each comparator is shown in Table I. Thus, even for $N_{k}=64$ the circuit in Fig. 8a will occupy the entire FPGA xc6slx45. Nevertheless, more advanced FPGAs allow the considered technique to be implemented.

Similarly blocks for discovering the maximum (the minimum) value in Fig. 4 (structures of these blocks are shown in Fig. 6) can be replaced with networks, which enable a group of maximum (minimum) values to be chosen. An example is shown in Fig. 9.

Let us consider the following 4 sorted subsets: 1) 126, 93, $11,6,3,2,1,0$; 2) 536, 40, 36, 35, 14, 14, 13, 8; 3) 512, 511, $507,468,321,169,15,3$; 4) 99, 97, 95, 93, 88, 22, 14, 12. We would like to find out a group of such $t$ values, in which the first $t / 2$ are the maximum values in all the sorted subsets and the second $t / 2$ are the minimum values in all the sorted subsets. 
a)

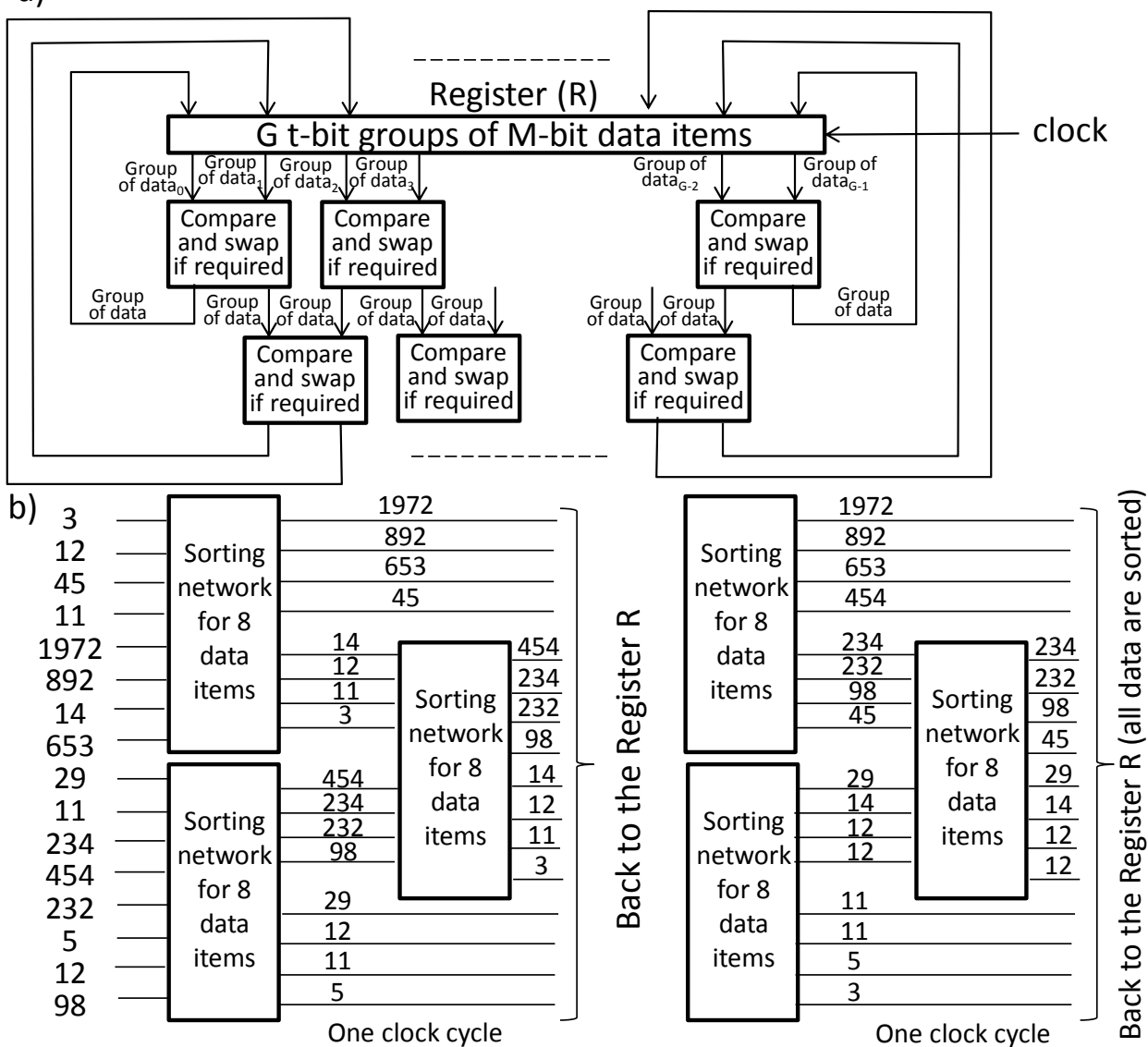

Fig. 8. Using sorting networks instead of comparators (a); an example of sorting (b).

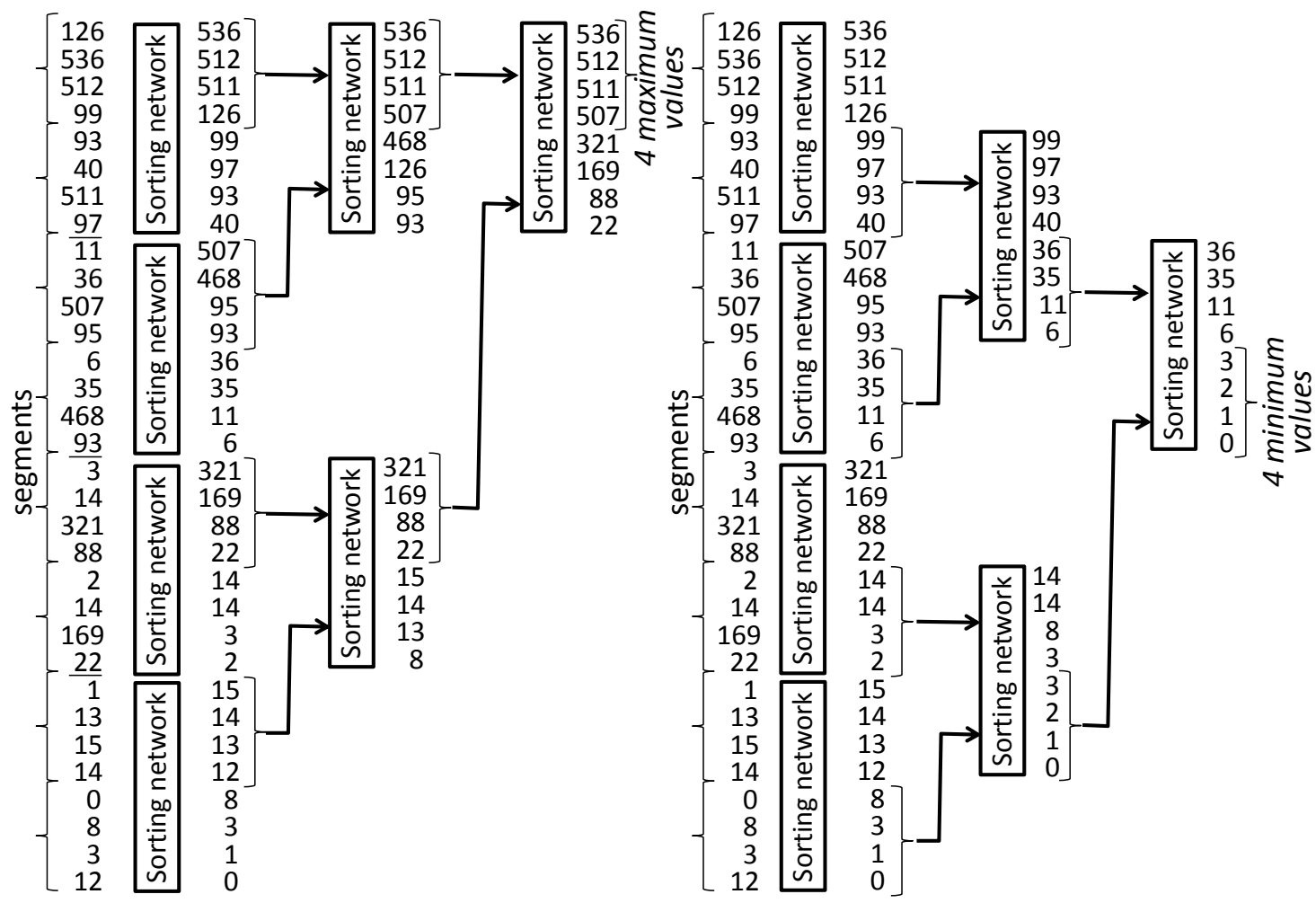

Fig. 9. Discovering groups with $t / 2$ maximum and $t / 2$ minimum values. 
Let us divide the sorted subsets in segments so that the first segment contains maximum values from all the sorted subsets, the second segment is composed of the next after the maximum values from all the sorted subsets, etc. For our example we will build the following 8 segments: 1) 126, 536, 512 , 99; 2) 93, 40, 511, 97; 3) 11, 36, 507, 95; 4) 6, 35, 468, 93; 5) $3,14,321,88$; 6) 2, 14, 169, 22; 7) 1, 13, 15, 14; 8) 0, 8, 3,12 .

Clearly, it is guaranteed that $\mathrm{h}$ left segments include $\mathrm{h}$ maximum values in entire set and $h$ right segments include $h$ minimum values in entire set. Now let us consider the segments instead of individual items in Fig. 6 ordering them with sorting networks as it is shown in Fig. 9. The circuit in Fig. 9 works much similar to the circuit in Fig. 6. The only difference is that segments are used instead of individual items. Now the circuit in Fig. 4 selects at each clock cycle 8 instead of 2 values and performance is increased 4 times. Note that since just a half of outputs are actually used in each sorting network in Fig. 9, the complexity of this sorting network (i.e., the number of comparators) can be reduced.

Now the registers $R_{\max }$ and $R_{\min }$ in Fig. 4 contain $t / 2$ segments and each segment is composed of $K$ values. The circuit in Fig. 4 executes the following steps:

1. Selecting in parallel (with the aid of the sorting networks described above) $t / 2$ maximum values from $R_{\max }$ and $t / 2$ minimum values from $R_{\min }$ with their indices (numbers of items in the respective register). Clearly, they are $t / 2$ largest and $t / 2$ smallest values in all the subsets.

2. Copying $t / 2$ maximum and $t / 2$ minimum values to the sorted set. At the same time replacing $t / 2$ maximum and $t / 2$ minimum values in the registers $R_{\max }$ and $R_{\min }$ with the next maximum and minimum segments from the sorted subsets. Both points 1 and 2 can be completed in one clock cycle.

3. The steps 1 and 2 are repeated until the final sorted set is built, which requires $\left(N_{0}+\ldots+N_{K-1}\right) / t$ clock cycles, i.e., $N / t$ for $N$ items in all the subsets.

\section{EXPERIMENTS AND COMPARISONS}

We found (see Section V) that step 2 (see Section II) can be used just for autonomous merging of a small number of sorted subsets. Thus, we have not analyzed this step in the experiments below.

Figure 10 presents the results of synthesis and implementation in different FPGAs (the types of FPGAs are indicated in Tables I and II) of circuits that sort small subsets of data (from 16 32-bit items to 1,024 32-bit items) using just step 1 (see Section II and Fig. 1a). Such circuits (Fig. 10) are extremely fast and can be used as components of real-time embedded systems implemented in the same microchip (an example of such system is given in [24]).

Figure 11 presents the results of simulation in software of circuits for complete data sorters (data sets up to $2^{14}$ of 32 data items were also verified in FPGAs; larger subsets were not synthesized and implemented in FPGAs due to very long synthesis and implementation time in ISE and Quartus; as an example we can say that obtaining the results in Table II for Altera FPGA and $N_{\max }=1024$ required about 2 hours in Quartus software executing in quad-core PC computer with frequency 3.2 GHz under 64-bit operating system).

Let us compare these results with the results in referenced publications. In [3] there are no data for sorting measured similar to Fig. $11 \mathrm{~b}$ and speed-up comparing to a Power PC 440 core is given. Thus, we will take data for median operators, which require less time than sorting of similar data. Processing median operators in FPGA of Virtex-5 family for large data sets requires approximately 19 ns per data item [3]. We can see from Fig. 11b that in our case this time is less than 5 ns. Let us compare now the results from Fig. 11 with sorting in GPU, which are given in Fig. 5 of [11]. Sorting of $16 \times 2^{20}$ data items requires between 500 and 1,000 milliseconds. In our case sorting $16 \times 2^{20}$ 32-bit data requires less than 80 milliseconds. Thus, the advantages of the proposed FPGAbased sorters are evident.

In [6] the maximum speed of sorting is estimated as 180 million records per second. Please note that this is an estimated result and even in this case it is worse than $5 \mathrm{~ns}$ per data item. Thus, our results are better because if we consider groups of items instead of individual items (see Fig. 11b) we can execute sorting with performance $3 \mathrm{~ns}$ per data item. Comparison with [8] (that provides very useful data for numerous sorting algorithms) also shows that the proposed data sorters are faster.

It should be also mentioned than in simulation results shown in Fig. 11 the clock frequency was considered to be just $50 \mathrm{MHz}$, which is less than real frequency in FPGA-based circuits. If we look at Table III we can see that actual achievable frequency is higher. Thus, we can expect better acceleration comparing to the results shown in Fig. 11b. Besides, potential improvements in Section VI are currently limited by not sufficient FPGA resources. Since such resources will undoubtedly be increased in upcoming generations of FPGA we can expect that the proposed methods will provide additional accelerations in future systems.

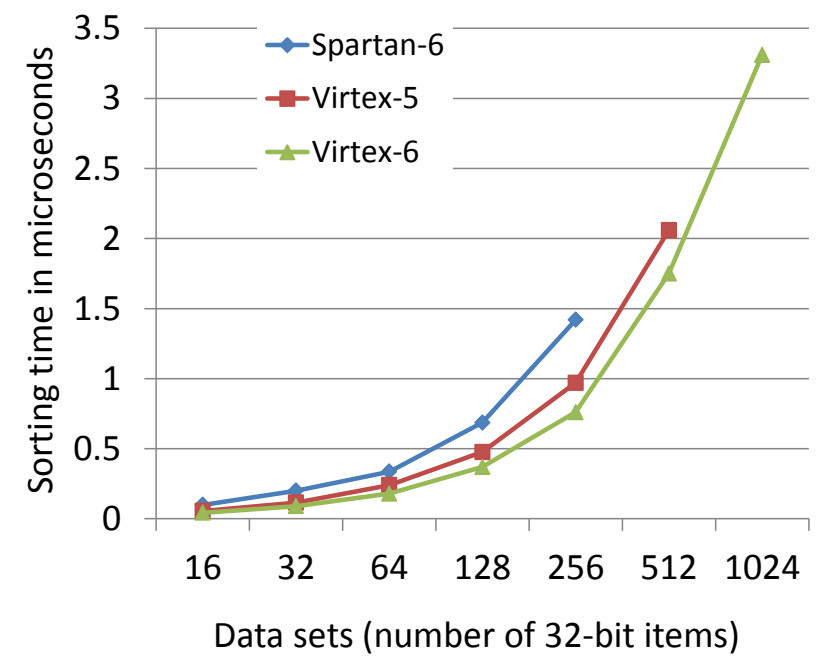

Fig. 10. The results of synthesis and implementations of data sorters for small sets of data items. 


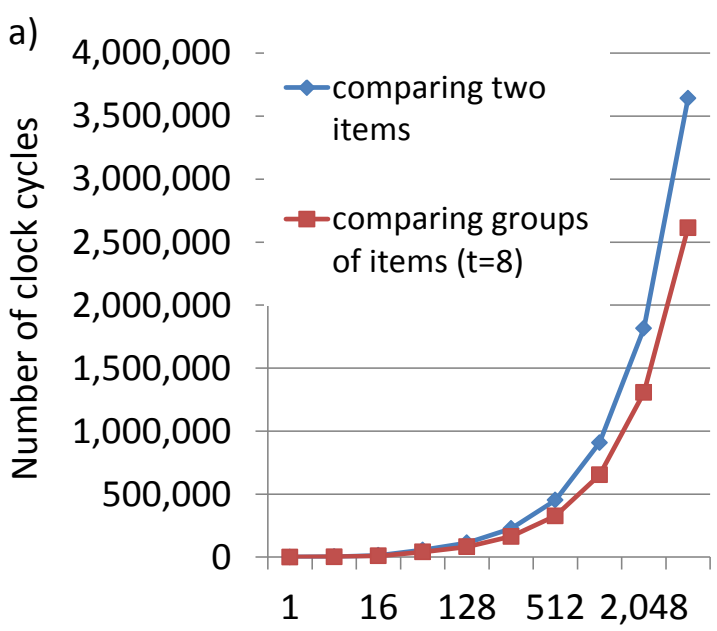

Data sets in $\mathrm{K}\left(\mathrm{K}=2^{10}\right) 32$-bit items

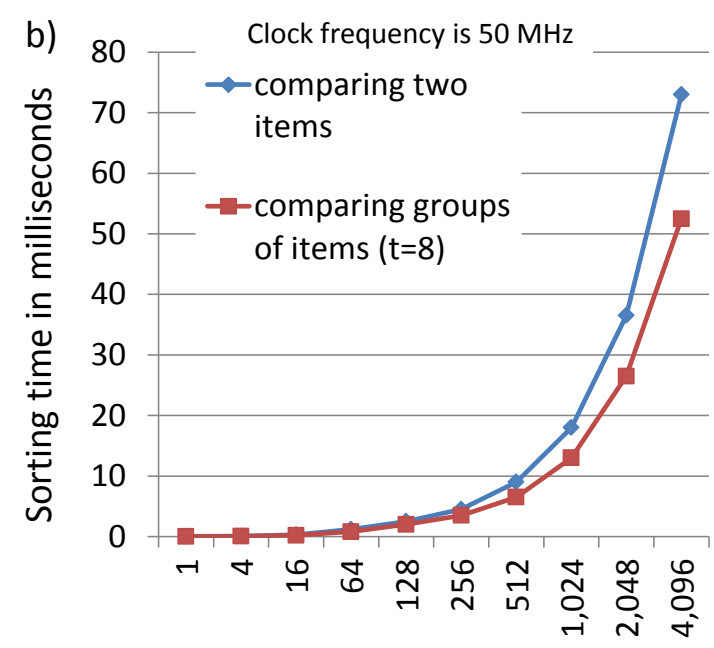

Data sets in $\mathrm{K}\left(\mathrm{K}=2^{10}\right) 32$-bit items

Fig. 11. The results of simulation in software of circuits for complete data sorters.

Another important feature of the proposed circuits is that the sorting time does not depend much on size of data items. To prove this conclusion we present in Fig. 12 the results of sorting similar to Fig. 10 but for two sizes of data items: 32 bits and 128 bits (the results for 128 bit data items are shown by dashed lines). Please note that we were able to implement in the FPGA xc5vlx110t of Virtex-5 family the data sorter for a set of 128 128-bit data and in [3] even 128 32-bit data items could not be sorted in the same FPGA due to the lack of FPGA resources.

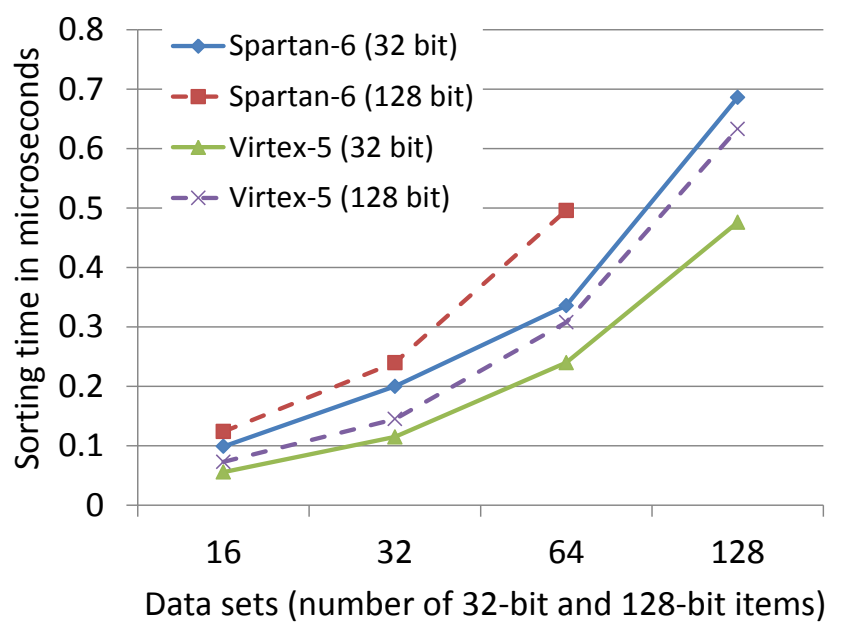

Fig. 12. The results of sorting similar to Fig. 10 but for 32- and 128-bit data items.

\section{CONCLUSION AND FUTURE WORK}

The paper suggests a new method and FPGA-based circuits for very fast parallel sorting of data. The method includes two basic steps: 1) representation of data in form of subsets in which data are sorted applying the proposed even-odd comparison-based networks, which combine parallel and sequential processing and allow the number of comparators to be reduced up to $N^{-1}$ ( $N$ is the number of data items to be sorted); 2) concurrent merging of many sorted subsets from the first step in a single sorted dataset with the aid of sequential extraction of minimal and maximal data items. The proposed circuits permit the number of clock cycles per data item to be significantly reduced. The technique enables datasets of size $N$ of $M$-bit items to be sorted in parallel in approximately $N$ clock cycles, which is faster than for other methods of sorting known from available publications. The first step can be executed autonomously for small datasets and in combination with the subsequent step. Experiments, which were done for up to $16,777,216\left(16 \times 2^{20}\right) 32$-bit data items, demonstrated very good results, in particular sorting was performed as fast as $3 \mathrm{~ns}-5 \mathrm{~ns}$ per data item with relatively low clock frequency, which was set to just $50 \mathrm{MHz}$.

A number of potential improvements were proposed but they were not completely verified in hardware and in software. The relevant task is considered to be one of future directions. Besides, due to very long synthesis and implementation time in commercial CAD systems, only sorters with up to $2^{14} 32$-bit items were verified in hardware and others (up to $2^{24} 32$-bit items) were modeled in software. We will also work on additional optimization of circuit components. A very interesting direction is software/hardware co-design when a problem of sorting is split between FPGA-based circuits and software of powerful processing systems. One possible way is to combine power of an ARM A9 dual-core Cortex processor and flexibility of Artix FPGA in the Zynq application-specific system-on-chip. This direction is also considered for the future.

\section{ACKNOWLEDGMENT}

This research was supported by the EU through European Regional Development Funds, by the institutional research funding IUT 19-1 of the Estonian Ministry of Education and Research, ESF grant 9251, and by Portuguese National Funds through FCT - Foundation for Science and Technology, in the context of the project PEst-OE/EEI/UI0127/2014. 


\section{REFERENCES}

[1] D. E. Knuth, The Art of Computer Programming. Sorting and Searching, vol. III. Addison-Wesley, 2011.

[2] T. H. Cormen, C. E. Leiserson, R. L. Rivest, and C. Stain, Introduction to Algorithms, $3^{\text {rd }}$ edition. MIT Press, 2009.

[3] R. Mueller, J. Teubner, and G. Alonso, "Sorting networks on FPGAs," The International Journal on Very Large Data Bases, vol. 21, no. 1, pp. 1-23, 2012. http://dx.doi.org/10.1007/s00778-011-0232-Z

[4] S. Chey, J. Liz, J. W. Sheaffery, K. Skadrony, and J. Lach, “Accelerating compute-intensive applications with GPUs and FPGAs," in 2008 Symposium on Application Specific Processors, 2008, pp. 101-107. http://dx.doi.org/10.1109/SASP.2008.4570793

[5] D. J. Greaves and S. Singh, "Kiwi: Synthesis of FPGA circuits from parallel programs," in 16th Int. Symp. on Field-Programmable Custom Computing Machines, 2008, pp. 3-12.

[6] R. D. Chamberlain and N. Ganesan, "Sorting on architecturally diverse computer systems," in 3rd Int. Workshop on High-Performance Reconfigurable Computing Technology and Applications, 2009, pp. 39-46.

[7] R. Mueller, "Data stream processing on embedded devices," Ph.D. thesis, ETH, Zurich, 2010.

[8] X. Ye, D. Fan, W. Lin, N. Yuan and P. Ienne, "High performance comparison-based sorting algorithm on many-core GPUs," in 2010 IEEE Int. Symp. on Parallel \& Distributed Processing, 2010.

[9] N. Satish, M. Harris, and M. Garland, "Designing efficient sorting algorithms for manycore GPUs," in 2009 IEEE Int. Symp. on Parallel \& Distributed Processing, 2009.

[10] D. Cederman and P. Tsigas, "A practical quicksort algorithm for graphics processors," in 16th Annual European Symp. on Algorithms, 2008, pp. 246-258.

[11] G. Gapannini, F. Silvestri, and R. Baraglia, "Sorting on GPU for large scale datasets: A throrough comparison," Information Processing and Management, vol. 48, no. 5, pp. 903-917, 2012. http://dx.doi.org/10.1016/j.ipm.2010.11.010

[12] P. Kipfer and R. Westermann, "Improved GPU sorting," in GPU Gems 2: Programming Techniques for High-Performance Graphics and General-Purpose Computation, M. Pharr and R. Fernando, Eds. Addison-Wesley, 2005. Available: http://http.developer.nvidia.com/ GPUGems2/gpugems2_chapter46.html.

[13] A. R. Brodtkorb, T. R. Hagen, and M. L. Sætra, "GPU programming strategies and trends in GPU computing," Journal of Parallel and Distributed Computing, vol. 73, no. 1, pp. 4-13, 2013. http://dx.doi.org/10.1016/j.jpdc.2012.04.003

[14] C. Grozea, Z. Bankovic, and P. Laskov, "FPGA vs. multi-core CPUs vs. GPUs," in Facing the Multicore-Challenge, R. Keller, D. Kramer, J. P. Weiss, Eds. Springer-Verlag, 2010, pp. 105-117. http://dx.doi.org/10.1007/978-3-642-16233-6 12

[15] M. Edahiro, "Parallelizing fundamental algorithms such as sorting on multi-core processors for EDA acceleration," in 14th Asia and South Pacific Design Automation Conference, 2009, pp. 230-233.

[16] B. Cope, P. Y. K. Cheung, W. Luk, and L. Howes, "Performance comparison of graphics processors to reconfigurable logic: A case study," IEEE Transactions on Computers, vol. 59, no. 4, pp. 433-448, 2010. http://dx.doi.org/10.1109/TC.2009.179

[17] J. Gonzalez and R. C. Núñez, "LAPACKrc: Fast linear algebra kernels/solvers for FPGA accelerators," Journal of Physics: Conference Series, vol. 180, 2009.

[18] S. Koehler, J. Curreri, and A. D. George, "Performance analysis challenges and framework for high-performance reconfigurable computing," Parallel Computing, vol. 34, no. 4-5, pp. 217-230, 2008. http://dx.doi.org/10.1016/j.parco.2008.01.008

[19] N. Moore, M. Leeser, and L. S. King, "VForce: An environment for portable applications on high performance systems with accelerators," Journal of Parallel and Distributed Computing, vol. 72, no. 9, pp. 1144-1156, 2012. http://dx.doi.org/10.1016/j.jpdc.2011.07.014
[20] M. Santarini, "Zynq-7000 EPP sets stage for new era of innovations," Xcell Journal, no. 75, 2011. [Online]. Available: http://www.eetimes.com/ design/programmable-logic/4217069/Zynq-7000-EPP-sets-stage-fornew-era-of-innovations.

[21] V. E. Alekseev, "Sorting algorithms with minimum memory," Kibernetica, vol. 5, pp. 99-103, 1969.

[22] I. Skliarova, V. Sklyarov, and A. Sudnitson, Design of FPGA-based Circuits using Hierarchical Finite State Machines. TUT Press, 2012.

[23] V. Sklyarov, I. Skliarova, D. Mihhailov, and A. Sudnitson, "Implementation in FPGA of address-based data sorting," in 21st Int. Conf. on FieldProgrammable Logic and Applications, 2011, pp. 405-410.

[24] V. Sklyarov and I. Skliarova, "Modeling, design, and implementation of a priority buffer for embedded systems," in 7th Asian Control Conf., 2009, pp. 9-14.

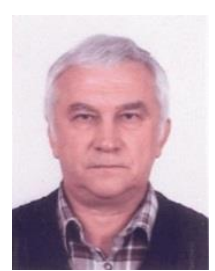

Valery Sklyarov received the engineering degree from the Technical University - UPI, Uljanovsk, Russia, in 1972, the Ph. D. degree in Computer Science from the Technical University - BSUIR, Minsk, Belarus, in 1978, the Doctor of Science degree in Computer Science from the Technical University - LETI, St. Petersburg, Russia, in 1986 and the Aggregation (Agregação) in Electronic Engineering from the University of Aveiro, Portugal, in 2001.

From 1972 to 1978 he was with the Research Institute, Minsk. From 1978 to 1994 he was with the Belorussian State University of Informatics and Radioelectronics, Belarus as an Associate Professor, and from 1987 onwards, as a Professor and the Head of the Computer Science Department. Since 1994 he has been with the Department of Electronics, Telecommunications and Informatics, University of Aveiro, Portugal, where he is currently a Professor of computer engineering. He has authored and co-authored 21 books and over 300 papers on subjects that include reconfigurable computing, data processing, digital design, computer architecture, and programming. The research interests include design and optimization of reconfigurable digital systems.

E-mail: sk1@ua.pt

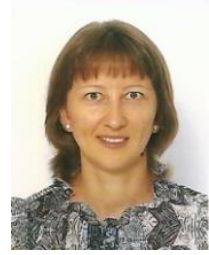

Iouliia Skliarova received the $\mathrm{M}$. Sc. degree in Computer Engineering from the Belorussian State University of Informatics and Radioelectronics, Minsk, Republic of Belarus, in 1998, and the Ph.D. degree in Electrical Engineering from the University of Aveiro, Portugal, in 2004

She is currently an Assistant Professor at the Department of Electronics, Telecommunications and Informatics, University of Aveiro. She has authored and co-authored four books and over 100 papers on subjects that include reconfigurable systems, data processing, digital design, and combinatorial optimization. Her research interests include reconfigurable digital systems, application-specific architectures, and computer-aided design.

E-mail: iouliia@ua.pt

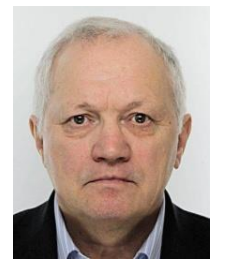

Alexander Sudnitson received the M. Sc. degree in Computer Engineering from Tallinn University of Technology, Tallinn, Estonia, in 1972, and the Ph. D. degree in Computer Engineering from the Institute of Cybernetics, Estonian Academy of Sciences in 1984.

$\mathrm{He}$ is an Associate Professor at the Department of Computer Engineering, Tallinn University of Technology, Estonia. His research interests include logic design, reconfigurable computing, and design automation. He has authored and co-authored over 100 peer-reviewed papers on subjects that include reconfigurable systems, digital design, asynchronous system design and computer-aided design.

$\mathrm{He}$ is a member of the IEEE.

E-mail: aleksander.sudnitson@ttu.ee 